\title{
A different interpretation of the annual and semiannual anomalies on the magnetic activity over the Earth
}

\author{
Francisco Azpilicueta ${ }^{1}$ and Claudio Brunini ${ }^{1}$ \\ Received 2 May 2012; revised 15 June 2012; accepted 18 June 2012; published 1 August 2012.
}

[1] The $\mathrm{H}$ component of the magnetic field measured at the terrestrial surface presents several periodic signals caused by changes in the ring current that flows within the terrestrial magnetosphere. One of the most important of them is associated to the phenomenon known as the Semiannual Anomaly which produces two significant minima during the equinoxes. This phenomenon is global, i.e., every observatory registers a similar effect independently of the hemisphere where it is located. A second important signal is due to the phenomenon known as the Annual Anomaly that produces significant different values for solstices, with a particular feature: the effect depends on the hemisphere where the observatory is located, with maximum during local summer. In spite of the time since their discoveries (more than a hundred years ago) the physical processes behind them are still open to discussion. In this work we present a new physical interpretation for the combined effects of both anomalies. The main concept developed is that along the year the shape of the magnetospheric cavities within which the ring current flows is deformed according to the geometric configuration between the solar wind and the magnetosphere.

Citation: Azpilicueta, F., and C. Brunini (2012), A different interpretation of the annual and semiannual anomalies on the magnetic activity over the Earth, J. Geophys. Res., 117, A08202, doi:10.1029/2012JA017893.

\section{Introduction}

[2] The magnetic field measured on the terrestrial surface has been systematically monitored and registered for about 170 years. Having this outstanding database is invaluable and the credits should go to the scientists and technicians that, as early as the middle of 19th century, acknowledged the importance of installing and maintaining their instruments as accurate as possible. Sometimes this meant keeping track of instruments located at places as distant as islands in the middle of the ocean. Credits should also go to those who digitalized the figures written on notebooks or registered on paper tapes and made the data series publicly available. The IAGA (International Association of Geomagnetism and Aeronomy) is a remarkable example of this.

[3] The geophysical variable measured at an observatory is the magnetic field at the particular place and at a particular moment. This means that the measurement is the resultant of the contribution of many different sources: global, regional, local, inner and outer ones, and all of them varying with time. The dominant contribution, $99 \%$, comes from the socalled main geomagnetic field produced at the terrestrial outer core (with a minor contribution from the crust). The

\footnotetext{
${ }^{1}$ Facultad de Ciencias Astronómicas y Geofísicas, Universidad Nacional de La Plata, CONICET, La Plata, Argentina.

Corresponding author: F. Azpilicueta, Facultad de Ciencias Astronómicas y Geofísicas, Universidad Nacional de La Plata, CONICET, Paseo del bosque s/n, 1900 La Plata, Argentina. (azpi@fcaglp.unlp.edu.ar)

(C)2012. American Geophysical Union. All Rights Reserved. 0148-0227/12/2012JA017893
}

remaining $1 \%$ is mainly of external origin, e.g., the magnetosphere and the ionosphere. The analysis of the long-term data series of representative magnetic observatories, like the four fundamental ones used to compute the Dst-index [Sugiura and Kamei, 1991], reveals a wide spectrum of signals: from periods of several decades, associated to the secular variation of the main field; passing through decadal periods produced by the solar activity and ending with the 27-day period signal due to the solar rotation. These last high-frequency signals are originated in the $1 \%$ external contribution and are generally referred to as magnetic activity.

[4] In between the previously mentioned signals there are two that have taken the attention of researchers since the beginning of this branch of scientific research [e.g., Sabine, 1856]. The first one is the widely known as the Semiannual Anomaly (SA) which produces two significant minimums during the equinoxes. This phenomenon is global, i.e., every observatory registers a similar effect independently of the hemisphere where it is located. As will be detailed in Section 3, in spite of the time since its discovery the physical process behind it is still an open issue. The second signal is the phenomenon known as the Annual Anomaly (AA) that produces significant different values for solstices, with a dependence on the hemisphere where the observatory is located. For example, a Northern observatory will register a value during June larger than during December. For a Southern observatory we will have the opposite case. The combination of both anomalies produces an annual pattern with their main features combined. For the SA there is a general consensus that is originated in a coupling process 


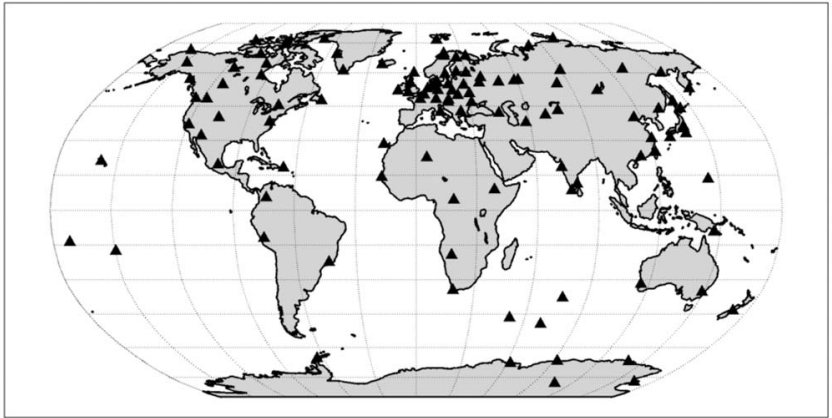

Figure 1. Geographical distribution of the magnetic observatories used. between the solar wind, the terrestrial magnetosphere and the ring current. For the AA there is not a consensus about its driving cause yet. It is worth to mention that these work focuses on the annual and semiannual anomalies caused by the ring current that flows within the terrestrial magnetosphere, but other similar anomalies are also found on magnetic index from mid- and high-latitudes.

[5] In this work we present a new interpretation of both phenomena, using as a working hypothesis that along the year the shape of the magnetospheric cavities within which the ring current flows is deformed according to the geometric configuration between the solar wind and the magnetosphere.

[6] Section 2 presents a description of the data used with details about the criteria applied to filter the original databases. Then it shows some examples of the annual patterns (including the SA and AA) of some magnetic observatories.

Table 1. List of the Observatories Sorted by Decreasing Latitude

\begin{tabular}{|c|c|c|c|c|c|c|c|c|c|c|c|}
\hline \# & ID & Lon (deg) & Lat (deg) & \# & ID & Lon (deg) & Lat (deg) & \# & ID & Lon (deg) & Lat (deg) \\
\hline 1 & $\mathrm{CCS}$ & 104.28 & 77.72 & 49 & DOU & 4.60 & 50.10 & 97 & PPT & -149.62 & -17.55 \\
\hline 2 & THL & -69.23 & 77.47 & 50 & LVV & 23.75 & 49.90 & 98 & TSU & 17.70 & -19.22 \\
\hline 3 & HRN & 15.55 & 77.00 & 51 & VIC & -123.42 & 48.52 & 99 & VSS & -43.65 & -22.40 \\
\hline 4 & $\mathrm{MBC}$ & -119.36 & 76.30 & 52 & NEW & -117.12 & 48.27 & 100 & GNA & 115.95 & -31.78 \\
\hline 5 & RES & -94.90 & 74.70 & 53 & FUR & 11.28 & 48.16 & 101 & HER & 19.23 & -34.42 \\
\hline 6 & DIK & 80.56 & 73.54 & 54 & CLF & 2.26 & 48.02 & 102 & $\mathrm{CNB}$ & 149.36 & -35.31 \\
\hline 7 & BRW & -156.62 & 71.32 & 55 & HRB & 18.19 & 47.87 & 103 & AMS & 77.57 & -37.83 \\
\hline 8 & TRO & 18.95 & 69.67 & 56 & STJ & -52.68 & 47.59 & 104 & EYR & 172.35 & -43.42 \\
\hline 9 & GDH & -53.53 & 69.25 & 57 & YSS & 142.72 & 46.95 & 105 & CZT & 51.87 & -46.43 \\
\hline 10 & CBB & -105.03 & 69.12 & 58 & THY & 17.90 & 46.90 & 106 & PAF & 70.22 & -49.35 \\
\hline 11 & ABK & 18.82 & 68.36 & 59 & ODE & 30.88 & 46.78 & 107 & AIA & -64.26 & -65.25 \\
\hline 12 & MMK & 33.08 & 68.25 & 60 & OTT & -75.55 & 45.40 & 108 & MIR & 93.02 & -66.55 \\
\hline 13 & SOD & 26.63 & 67.37 & 61 & MMB & 144.19 & 43.91 & 109 & DRV & 140.01 & -66.67 \\
\hline 14 & $\mathrm{CMO}$ & -147.86 & 64.87 & 62 & VLA & 132.17 & 43.68 & 110 & MAW & 62.88 & -67.60 \\
\hline 15 & BLC & -96.03 & 64.33 & 63 & AAA & 76.92 & 43.25 & 111 & SBA & 166.78 & -77.85 \\
\hline 16 & LRV & -21.70 & 64.18 & 64 & PAG & 24.18 & 42.52 & 112 & VOS & 106.87 & -78.45 \\
\hline 17 & YKC & -114.47 & 62.47 & 65 & AQU & 13.32 & 42.38 & & & & \\
\hline 18 & YAK & 129.72 & 62.02 & 66 & TFS & 44.70 & 42.08 & & & & \\
\hline 19 & POD & 90.00 & 61.40 & 67 & TKT & 69.62 & 41.33 & & & & \\
\hline 20 & NAQ & -45.43 & 61.16 & 68 & BOU & -105.24 & 40.14 & & & & \\
\hline 21 & NUR & 24.66 & 60.51 & 69 & BJI & 116.18 & 40.06 & & & & \\
\hline 22 & MGD & 151.02 & 60.12 & 70 & FRD & -77.37 & 38.20 & & & & \\
\hline 23 & LER & -1.18 & 60.05 & 71 & ASH & 58.11 & 37.95 & & & & \\
\hline 24 & LNN & 30.70 & 59.95 & 72 & FRN & -119.72 & 37.08 & & & & \\
\hline 25 & LOV & 17.83 & 59.35 & 73 & KAK & 140.19 & 36.23 & & & & \\
\hline 26 & FCC & -94.09 & 58.76 & 74 & KNZ & 139.97 & 35.25 & & & & \\
\hline 27 & BOX & 38.97 & 58.03 & 75 & HTY & 139.83 & 33.07 & & & & \\
\hline 28 & SIT & -135.33 & 57.07 & 76 & TUC & -110.83 & 32.25 & & & & \\
\hline 29 & SVD & 61.07 & 56.73 & 77 & KNY & 130.88 & 31.42 & & & & \\
\hline 30 & ARS & 58.57 & 56.43 & 78 & $\mathrm{SSH}$ & 121.19 & 31.10 & & & & \\
\hline 31 & RSV & 12.45 & 55.85 & 79 & TEN & -16.27 & 28.48 & & & & \\
\hline 32 & KZN & 48.85 & 55.83 & 80 & LNP & 121.17 & 25.00 & & & & \\
\hline 33 & BFE & 11.67 & 55.63 & 81 & GZH & 113.34 & 23.09 & & & & \\
\hline 34 & MOS & 37.32 & 55.47 & 82 & TAM & 5.53 & 22.80 & & & & \\
\hline 35 & ESK & -3.20 & 55.32 & 83 & HON & -158.00 & 21.32 & & & & \\
\hline 36 & NVS & 82.90 & 55.03 & 84 & TEO & -99.18 & 19.75 & & & & \\
\hline 37 & MEA & -113.35 & 54.62 & 85 & ABG & 72.87 & 18.64 & & & & \\
\hline 38 & HLP & 18.82 & 54.61 & 86 & SJG & -66.12 & 18.38 & & & & \\
\hline 39 & MNK & 27.88 & 54.50 & 87 & MBO & -16.96 & 14.39 & & & & \\
\hline 40 & WNG & 9.07 & 53.75 & 88 & GUA & 144.87 & 13.58 & & & & \\
\hline 41 & PET & 158.25 & 52.97 & 89 & ANN & 79.68 & 11.37 & & & & \\
\hline 42 & WIT & 6.67 & 52.82 & 90 & $\mathrm{AAE}$ & 38.77 & 9.03 & & & & \\
\hline 43 & IRT & 104.45 & 52.17 & 91 & TRD & 76.95 & 8.48 & & & & \\
\hline 44 & NGK & 12.68 & 52.07 & 92 & FUQ & -73.74 & 5.47 & & & & \\
\hline 45 & VAL & -10.25 & 51.93 & 93 & BNG & 18.57 & 4.44 & & & & \\
\hline 46 & BEL & 20.79 & 51.84 & 94 & PMG & 147.15 & -9.40 & & & & \\
\hline 47 & HAD & -4.48 & 50.99 & 95 & HUA & -75.33 & -12.05 & & & & \\
\hline 48 & KIV & 30.30 & 50.72 & 96 & API & -171.78 & -13.81 & & & & \\
\hline
\end{tabular}



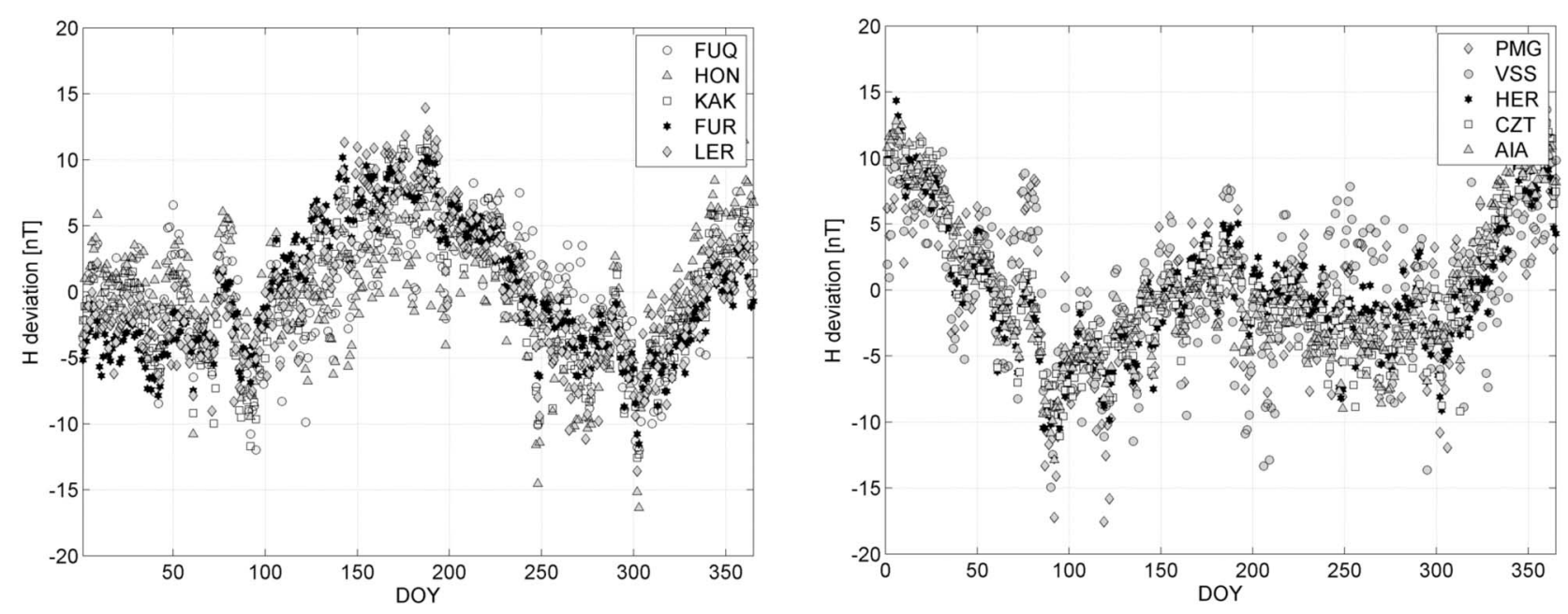

Figure 2. Annual patterns for (left) five northern and (right) five southern observatories.

Based on the patterns obtained in Section 2, Sections 3 and 4 describe with details the distinguishable features of the each of the anomalies. Section 5 introduces the new concept of a linear dependence between the measured magnetic activity and the geographic latitude of the observatory. Section 6 presents a model proposed by Malin and Isikara [1976] which could help to physically understand the annual anomaly and Section 7 presents an interpretation of the results of Section 5 and the Malin-Isikara model. Finally, Section 8 summarizes the results and highlights the important findings of our work.

\section{H Magnetic Component Data Series}

[7] The data used in this work are the horizontal component (H-component) of the magnetic field measured at IAGA observatories (http://www.iugg.org/associations/iaga.php). The selected observatories are included in the Geomagnetic Data Master Catalogue, and the data series are publicly available at the server of the World Data Centre for Geomagnetism, Edinburgh (http://www.wdc.bgs.ac.uk/catalog/ master.html).

[8] A rigorous data filtering criteria was applied to warrant the quality of the data used: i) only days with 24 hourly measurements were included; ii) only years with at least 330 daily measurements were included; iii) those years that presented a sudden jump in the data, clearly not associated to a natural process but to any problem with the instrument were excluded; and iv) only observatories with at least 15 years of data (after applying the other filters) were included. The filtering process left 112 observatories to work with. Figure 1 shows the geographical distribution of these observatories and Table 1 lists the names and the geographic coordinates of them. Although the latitudinal distribution is quite inhomogeneous, the Southern observatories covered the same latitudinal range as the Northern observatories. It is worth to mention that the four fundamental observatories involved in the computation of the Dst-index [Sugiura and Kamei, 1991], HON (Honolulu, USA), KAK (Kakioka, Japan), HER (Hermanus, South Africa) and SJG (San Juan, Puerto Rico) are included in this work.
[9] The data series for the 112 observatories were processed following the technique described in Azpilicueta et al. [2012]. The basic steps behind this technique are: i) daily mean values are computed reducing the database to one value per day; ii) for each year of data, a linear trend is adjusted and subtracted thus obtaining for each observatory a series of deviations of the H-component (H-deviations) from the value expected according to the linear trend; iii) finally an annual characteristic pattern for each observatory is obtained by computing the mean deviation for each DOY (day of the year).

[10] This technique is useful for reconstructing the annual pattern without previous assumptions on the periodicity of the resulting signals. The interested reader is referred to the previous reference for details. Figure 2 shows examples of the annual patterns so obtained. Figure 2 (left) shows the annual patterns from five Northern observatories while Figure 2 (right) shows five Southern ones.

\section{Semiannual Anomaly}

[11] The main features coming from both panels of Figure 2 are the two significant minimums occurring at approximately DOYs 90 and 280 (close to the Equinoxes) on the 10 patterns presented. These quasi-equinoxial minima are the manifestation of the SA. The existence of the SA has been known for about 170 years and it is widely accepted within the aeronomic community that it reflects changes on the intensity of the ring current that flows around the Earth. There is also a large consensus that the driving mechanism behind these variations is associated to a coupling process between the solar wind and magnetosphere. During the 20th century researchers have systematically observed the SA and spent efforts to understand the physical mechanism behind it.

[12] The historical benchmarks associated to the development of new theories on this anomaly are: i) the "axial hypothesis" proposed by Cortie [1912], which has the heliographic latitude of the Earth as the driven parameter; ii) the "equinoctial hypothesis" that was first proposed by Bartels [1932], in which the driven parameter is the tilt angle between the Sun-Earth direction and the geomagnetic 


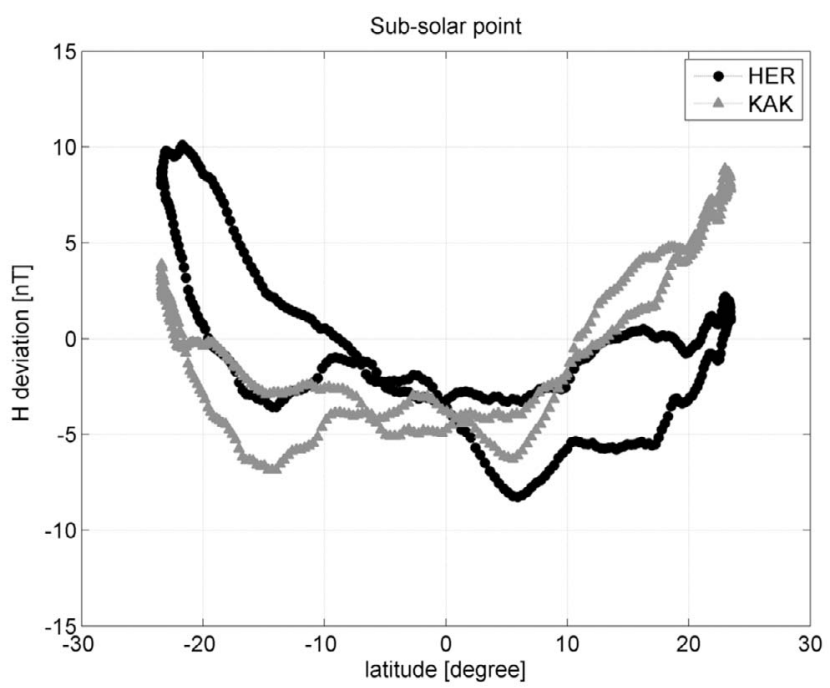

Figure 3. Annual patterns for KAK (Northern Hemisphere gray triangles) and HER (Southern Hemisphere - black circles) mapped against the latitude of the sub-solar point. A 20-day moving average filter was applied to reduce the dispersion of the data.

dipole; iii) the "Russell and McPherron hypothesis" presented in Russell and McPherron [1973], in which the main parameter is the southward component of the interplanetary magnetic field. In spite of all these efforts a conclusive explanation has remained elusive until the present.

[13] From Figure 2 is seen that the minima occur simultaneously on the 10 observatories, thus verifying that the SA is on-phase on both hemispheres. Another interesting feature is that for the Southern observatories the March minimum appears lower than the observed for the Northern ones. The September minimum shows the opposite behavior. A list of works dealing with the SA would be large; as a representative sample we can mention, e.g., Chaman Lal [1998], Clúa de Gonzalez et al. [2001], O'Brien and McPherron [2002], Lyatsky and Tan [2003], Häkkinen et al. [2003], Cliver et al. [2004], Mursula et al. [2011], and Svalgaard [2011].

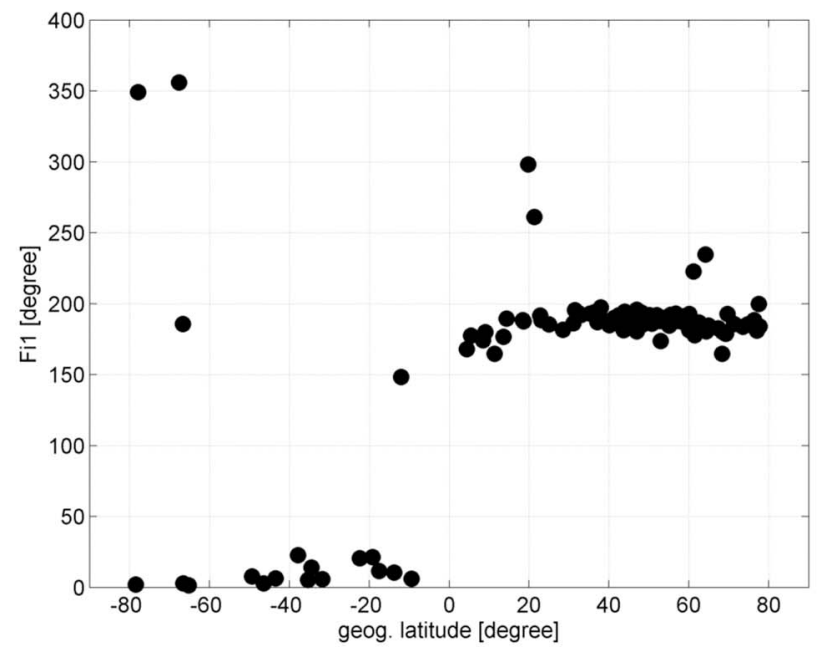

[14] Figure 3 shows the annual patterns from KAK and HER observatories mapped against the latitude of the subsolar point (i.e., the solar declination). The patterns from both observatories present an U-shape, approximately centered for sub-solar point latitude equals to zero (equinoxes), and with the extremes corresponding to sub-solar point latitude equals to $\sim \pm 23^{\circ}$ (June and December solstices). This is a convenient way of representation since as was mentioned at the second paragraph of this section the angle between the sub-solar point and the dipole axis is one of the proposed drivers for the phenomenon. It is worth to mention that the daily average of the direction of the magnetic dipole coincides with the terrestrial rotation axis. Then, the angle between the solar direction and the terrestrial rotation axis becomes the driven parameter for daily basis studies. This is equivalent to say that the driving parameter is the solar declination.

\section{Annual Anomaly: Inter-hemispheric Asymmetry on the Annual Patterns}

[15] Looking at Figure 2 and comparing Figure 2 (left) with Figure 2 (right) a second important characteristic of the patterns that comes out is the inter-hemispheric asymmetry: the Northern observatories show June maxima significantly and systematically larger than the corresponding Southern observatories maxima. The December maxima show the opposite behavior. These different behaviors between the solstices are attributed to the AA. This issue has been studied and documented, a list of representative works could include Malin and Isikara [1976], Stening and Winch [1987], Mayaud [1978], Le Mouël et al. [2004], and Wardinski and Mandea [2006].

[16] On Figure 3 the effect of the annual anomaly is manifested with HER values (circles) larger than the KAK ones (triangles) for the period when the latitude of the subsolar point is close to $-23^{\circ}$ (December solstice) and KAK values larger than HER's when the latitude of the sub-solar point is close to $+23^{\circ}$ (June solstice).

[17] The behavior described in the previous paragraph led several researchers (see references above) to propose a second

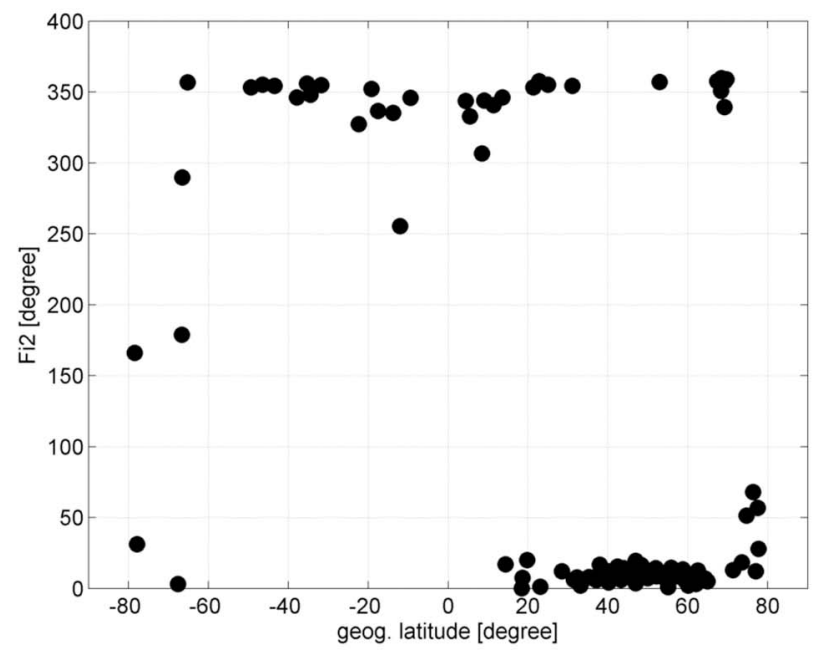

Figure 4. (left) The adjusted $\varphi_{1}$ (annual harmonic) and (right) the adjusted $\varphi_{2}$ (semiannual harmonic) mapped against the geographic latitude of the observatory. 

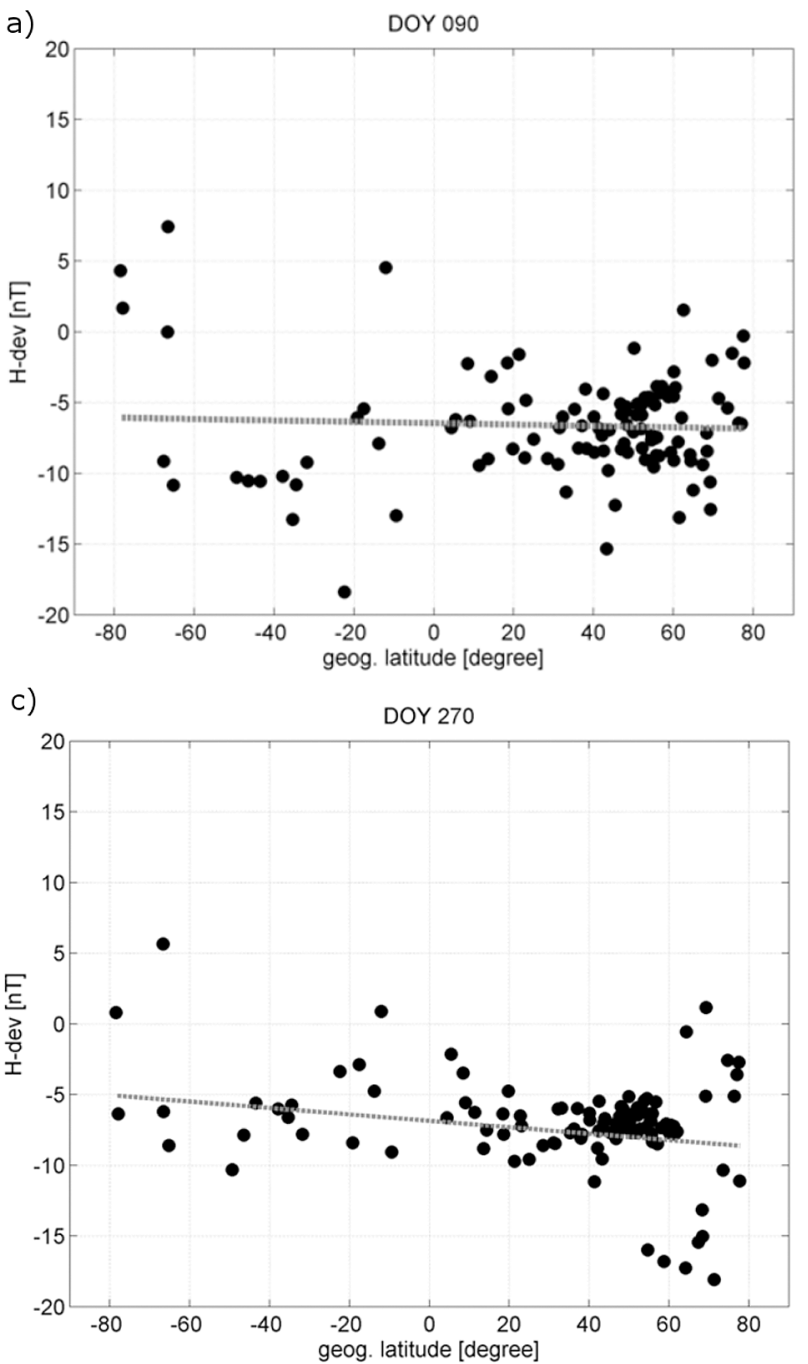

b)

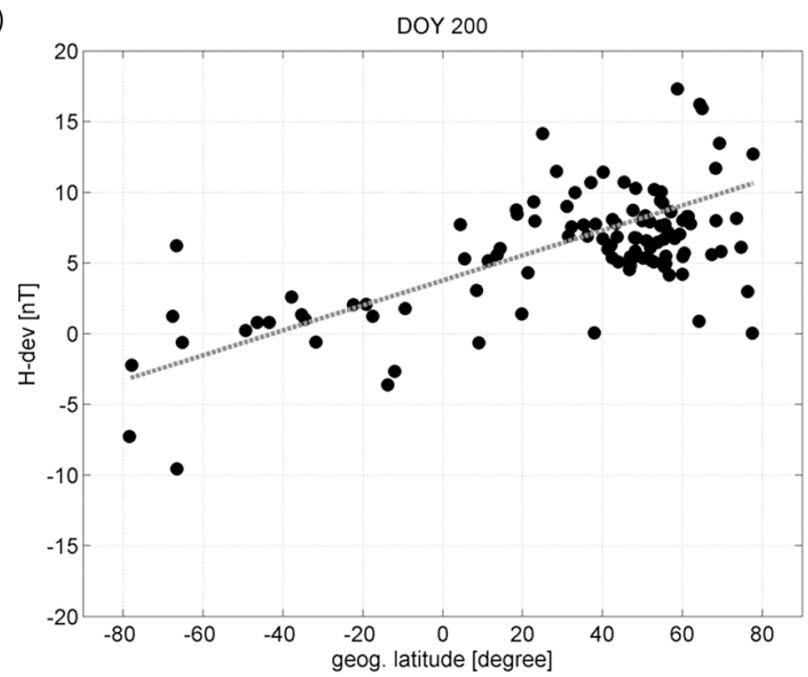

d)

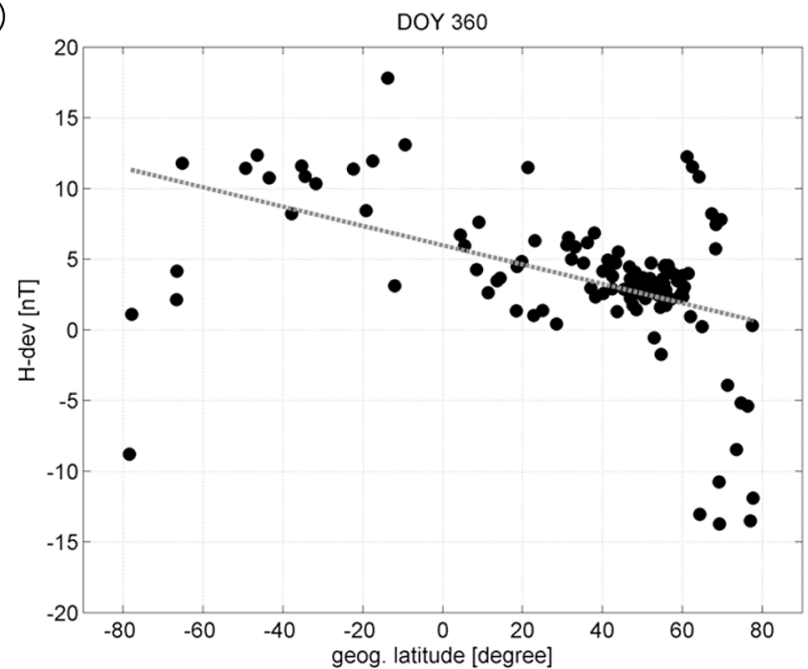

Figure 5. The dots represent the H-deviation mapped against the geographic latitude of the observatories for DOYs (a) 090, (b) 200, (c) 279, and (d) 300. The line represents the linear fit for each day.

anomaly (with annual period) over imposed to the semiannual anomaly. Following this ideas, a function given by $v(D O Y)=A \cos \left(\frac{2 \pi}{365} \cdot D O Y+\varphi_{1}\right)+B \cos \left(\frac{\pi}{365} \cdot D O Y+\varphi_{2}\right)$, where $v$ stands for the mean H-deviation, was applied to the database adjusting by a least squares method the four parameters: $A, \varphi_{1}, B, \varphi_{2}$. Figure 4 shows the adjusted $\varphi_{1}$ and $\varphi_{2}$ mapped against the geographic latitude of the observatories.

[18] From Figure 4 (left) it is evident that the annual variation is on phase opposition between both hemispheres. Figure 4 (right) serves to confirm that the semiannual anomaly is on-phase at a global scale. It is worth to recall that a phase close to $0^{\circ}$ is equivalent to a phase close to $360^{\circ}$.

\section{Correlation Between the H-Deviation and the Latitude of the Observatories}

[19] An interesting result appears when the H-deviations are mapped against the geographic latitude of the observatories on a daily basis. Figure 5 shows four of this graphics, corresponding to DOYs: 090, 200, 270 and 360 (approx. the equinoxes and solstices). The dots correspond to the $\mathrm{H}$-deviations while the strait line corresponds to the linear fit to the data of the day. Analyzing the sequence it comes out that for the equinoxes the lines appear with slopes close to zero and with intercepting values (values for latitude equals to zero) negative and reaching the minimum; for the June solstice the intercepting value is positive with positive slope; and for the December solstice the intercepting value is also positive but the slope is negative. This preliminary analysis suggested that the intercepting values could be associated to the SA while the slopes could reflect the effect of the AA.

[20] For further analyzing the previous hypothesis, a linear fit was computed for every DOY. Figure 6 (left) shows the sequence of the intercept value against DOY and represents the values that would measure an 'ideal' observatory located at the geographic equator along the year. This term retains the general structure of the SA observed at each observatory but corrected from the annual variation. Figure 6 (right) shows the behavior of the slopes against DOY. It is interesting to see that it presents a smooth variation along the year and seems to correlate with the latitude of the sub-solar 

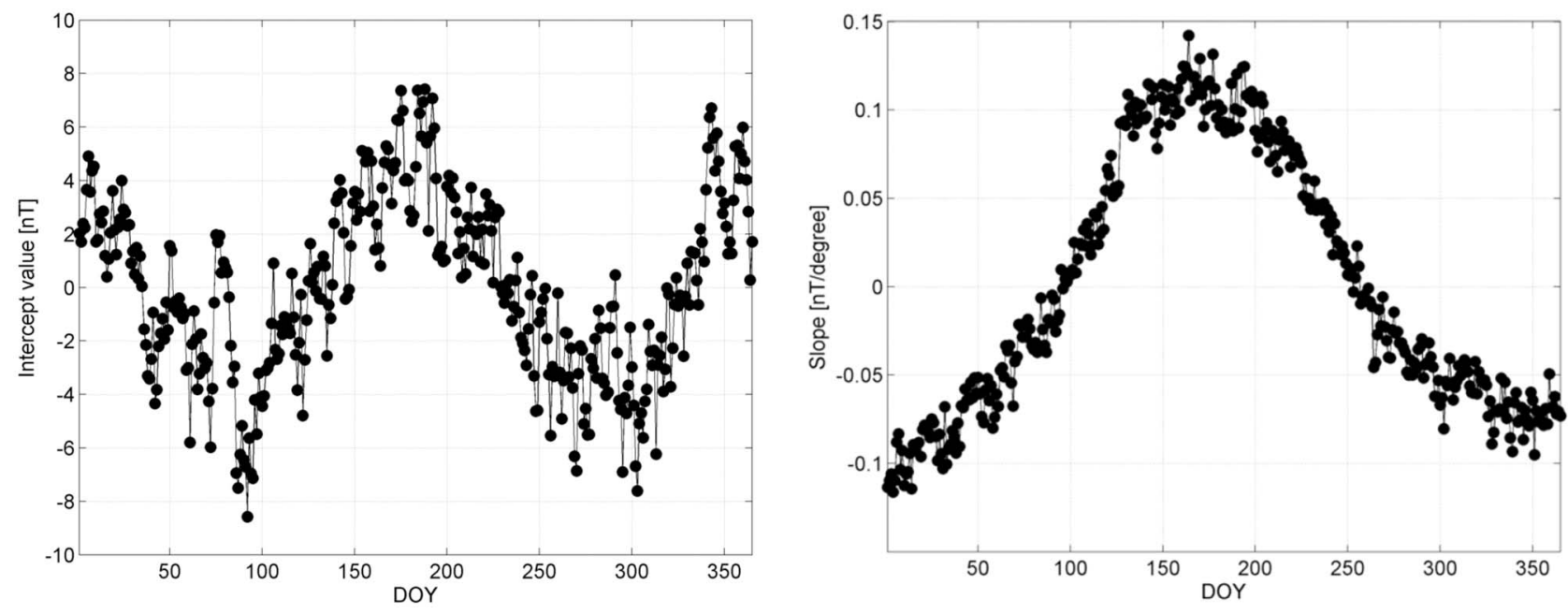

Figure 6. Intercept value and slope for the daily linear fits as function of the DOY.

point. In Section 6 we will return to these issues and analyze the results in the theoretical framework presented in Section 5.

[21] To close this section it is worth to mention that the erratic behavior of the H-deviation on the panels of Figure 5 for the high latitude regions, |geog. latitude $\mid>60$, can be attributed to two sources. The first one is that the $\mathrm{H}$-component at high latitudes is less representative of the intensity of the ring current, since it would be expected that the magnetic field induced by the ring current would be approximately parallel to the rotation axis. The second source could be a contribution to the magnetic activity due to the auroral electro-jet.

\section{The Malin-Isikara Model}

[22] Malin and Isikara [1976] studied the annual variation of the geomagnetic field measured at ground stations, explicitly understanding by annual variation a phenomenon with a 1-year period, thus excluding from analysis the semiannual anomaly. In that work the authors used magnetic determinations from 69 observatories with a complete longitudinal coverage and the best Southern hemisphere coverage available, and a time span going from 1957 to 1961. In this pioneering work Malin and Isikara proposed a model for the interaction between the solar wind and the magnetosphere that could produce an annual variation on the measurements made at the geomagnetic observatories, with phase-opposition between hemispheres.

[23] The most significant elements of the Malin-Isikara idea are: i) they assumed a solar wind radially flowing from the Sun; ii) the locus where the solar wind exerts the maximum dynamic pressure over the terrestrial magnetosphere (which we called SW point) is on the Sun-Earth direction, at some few radii from the geocenter; iii) as the Sun-Earth direction makes it annual excursion, the latitude of the SW varies from $+23^{\circ}$ (June) to $-23^{\circ}$ (December) and results in changes of the geometrical configuration between the SW
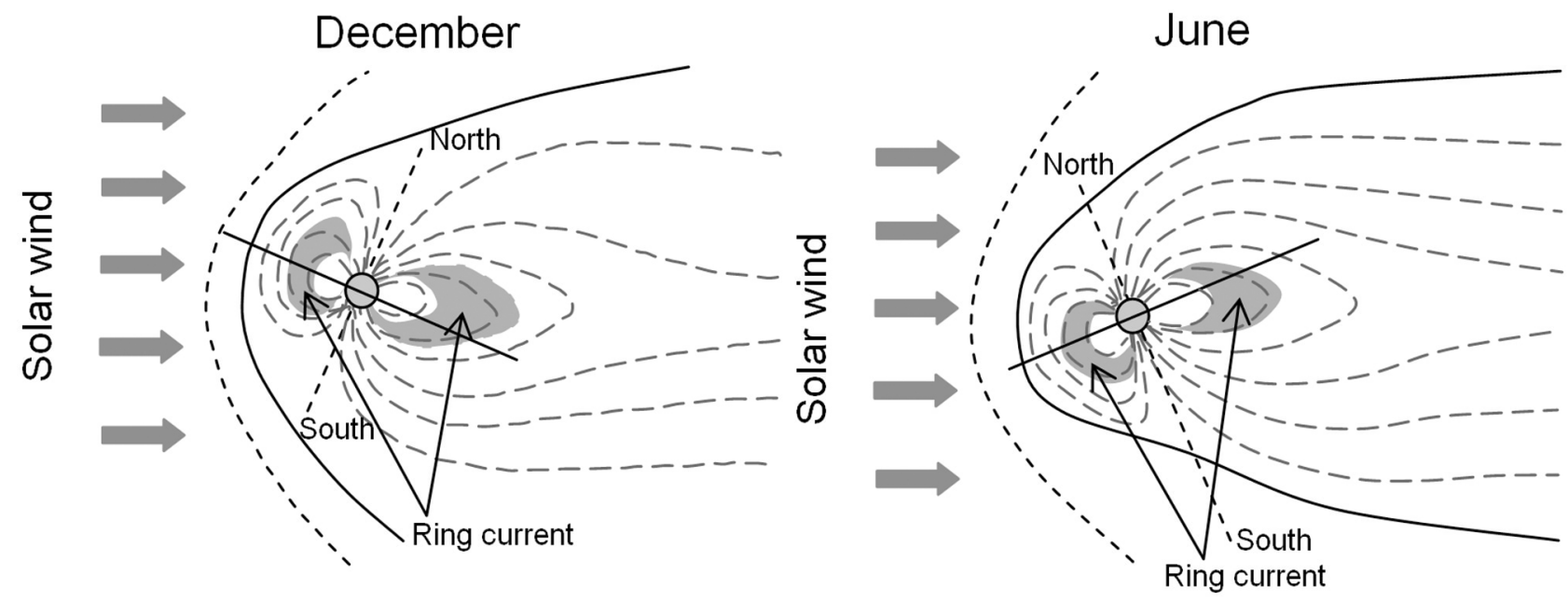

Figure 7. Schematic showing the Malin-Isikara effect (following on Malin and Isikara [1976]). (left) The Northern hemisphere winter configuration, when the ring and tail currents are displaced northward. (right) The Northern summer, with the ring and tail currents displaced southward. 

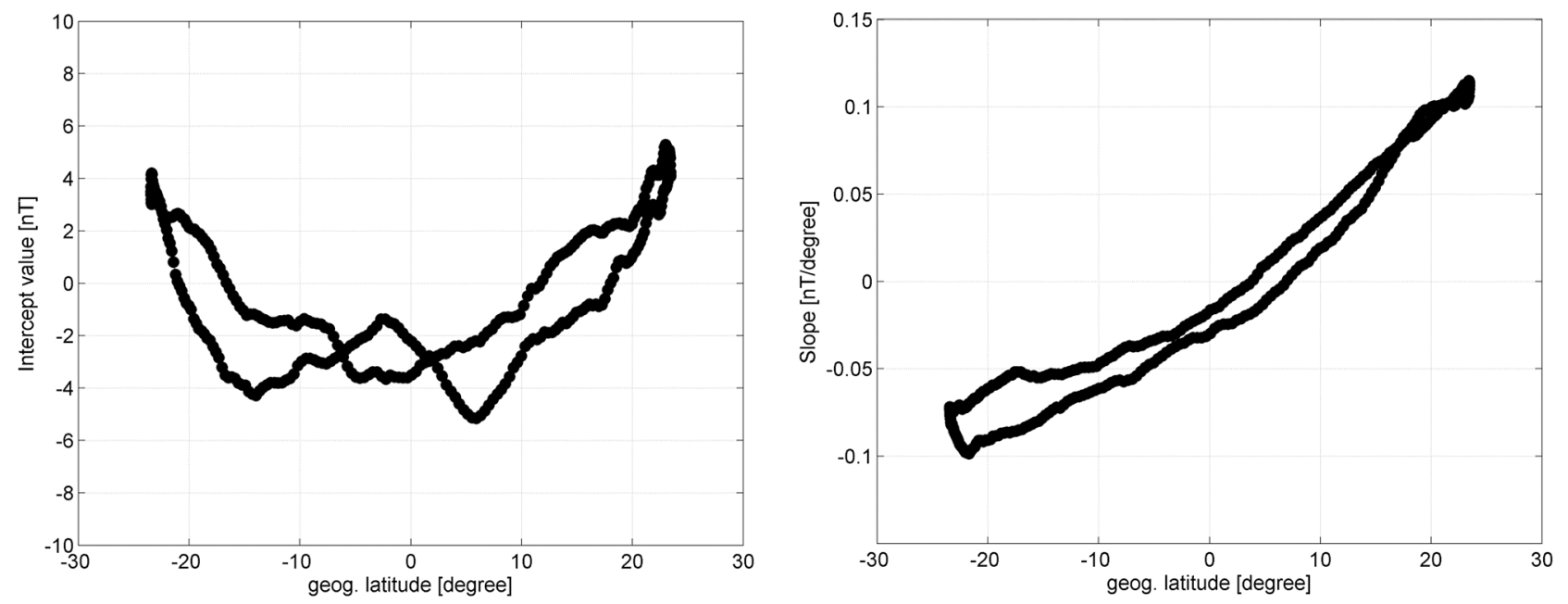

Figure 8. (left) The intercept and (right) the slopes mapped against the geographic latitude of the subsolar point.

and the force lines of the geomagnetic field, completing a cycle after one year.; iv) the different geometrical configurations produce deformations on the shape of the magnetospheric cavities within which the ring current flows.

[24] The extreme situations of the effect occur during the solstices: the compression over the hemisphere where the Sun is results on asymmetric cavities at each hemisphere (see Figure 7). This deformation causes an asymmetric distribution of the ring and tail currents between the hemispheres. Then Malin-Isikara model predicts that during June (Northern hemisphere summer) the currents should be shifted to the Southern hemisphere and thus the induced magnetic field measure over Northern Hemisphere observatories should be reduced then causing an increase on the measured magnetic field. The opposite effect occurs during December, since during this month the ring and tail currents are shifted to the Northern hemisphere and then the magnetic activity induced by the ring is increased and should produce a decrease on the measured magnetic field over Northern observatories. For Southern observatories we will have the opposite situation.

[25] The Malin-Isikara model predicts an annual variation of the magnetic activity, induced by the ring current, which phase depends on the latitude of the observatory. These properties fit quite well to the results presented in Section 5.

\section{Analysis and Interpretation}

[26] The purpose of this section is to interpret the results obtained in Section 5 within the framework of the MalinIsikara model described in Section 6. In principle we can make the hypothesis that the annual pattern (including the SA and AA) observed at a particular observatory is the result of two effects acting simultaneously. The first one is associated to a still unknown mechanism (recall the second paragraph of Section 3) which produces a seasonal modulation on the intensity of the ring current that induces a similar seasonal modulation on the magnetic activity at the observatory. The second phenomenon is due to the MalinIsikara effect and causes the magnetic activity measured at the terrestrial surface to depend on the latitude of the observatory and on the moment of the year (i.e., on the DOY). Then, for example during June, the seasonal modulation would have a value approximately given by the intercept value (Figure 6, right), but the ring current would be shifted to the Southern Hemisphere. So the result would be that during June the value measured at Northern (Southern) observatories would be higher (lower) than the expected one. Stening and Winch [1987] has already noted the necessity of these two effects.

[27] Finally, Figure 8 depicts the intercept values (Figure 8, left) and the slopes (Figure 8, right) mapped against the latitude of the sub-solar point, following the scheme of Figure 3.

[28] The analysis of Figure 8 (left) indicates that the correlation between the intercept values and the sub-solar latitude is significant, with minima during periods with latitudes close to zero (equinoxes), and maximums for the maximum departures of the sub-solar point from the Equator (solstices). This was already found when inspecting Figure 3, but in this graphic the effect of the annual variation is canceled. In our approach the effect of the annual variation is captured by the slope which, according to Figure 8 (right) is highly linearly correlated with the SW (sub-solar) point latitude.

[29] A second order mechanism seems to be present on both graphics of Figure 8 , in the sense that there seems to be a second effect that could lead to slightly different results for going and returning paths from/to an equinox to/from a solstices. Presently we are studying to possibility to include to the variation of the Sun-Earth distance along the year to explain this phenomenon. Another hypothesis we are studying is a non-radial solar wind. The results obtained are encouraging and we hope to publish them soon.

\section{Conclusions}

[30] Based on a wide and rigorously selected database, we reconstructed the annual patterns caused by the ring current on the H-component at each of 110 IAGA observatories. 
These annual patterns reflect the combined effect of the Semiannual and the Annual Anomalies registered at each observatory.

[31] From the analysis of the daily behavior of the $\mathrm{H}$-deviation, we found a significant linear correlation between the measured H-deviation and the observatory geographic latitude. Extending the fundamental concepts of the Malin-Isikara model for the interaction between the solar wind and the terrestrial magnetosphere, we proposed a new manner to interpret the physical meaning of the Annual Anomaly as a modulating effect on the Semiannual Anomaly (seasonal) effect.

[32] According to this interpretation the magnetic activity registered at an observatory would depend on the DOY and on the latitude of the observatory. In other words, the registered magnetic activity depends on two main variables. The first one is the latitude of the direction where the solar wind exerts the maximum dynamic pressure over the terrestrial magnetosphere. This direction establishes the intensity level of the Semiannual Anomaly and also the deformation on the magnetospheric cavities within which the ring current flows. The second variable is the latitude of the observatory, because with an irregular spatial distribution of the ring current, the measured magnetic activity would depend on the relative position between the observatory and the current.

[33] The agreement between the results and what would be expected (see Figure 8 and the associated discussion) is quite satisfying and at the same encouraging to continue our work.

[34] Acknowledgments. The authors thank the IAGA (International Association of Geomagnetism and Aeronomy) and the World Data Centre for Geomagnetism, Edinburgh, for providing the outstanding data series used in this work. The authors thank an anonymous reviewer and M. Fujimoto for their valuable comments about our work.

[35] Masaki Fujimoto thanks the reviewers for their assistance in evaluating this paper.

\section{References}

Azpilicueta, F., C. Brunini, and E. Camilión (2012), The geomagnetic semiannual anomaly on the four Dst-fundamental observatories: Dependences with Sun-Earth physical parameters, J. Geophys. Res., 117, A07204, doi:10.1029/2012JA017730.
Bartels, J. (1932), Terrestrial-magnetic activity and its relations to solar phenomena, J. Geophys. Res., 37, 1-52, doi:10.1029/TE037i001p00001.

Cliver, E. W., L. Svalgaard, and A. G. Ling (2004), Origins of the semiannual variation of geomagnetic activity in 1954 and 1996, Ann. Geophys., 22, 93-100, doi:10.5194/angeo-22-93-2004.

Clúa de Gonzalez, A. L., V. M. Silbergleit, W. D. Gonzalez, and B. T. Tsurutani (2001), Annual variation of geomagnetic activity, J. Atmos. Sol. Terr. Phys., 63, 367-374, doi:10.1016/S1364-6826(00)00190-5.

Cortie, A. L. (1912), Sunspots and terrestrial magnetic phenomena, 1898-1911: The cause of the annual variation in magnetic disturbances, Mon. Not. R. Astron. Soc., 7(3), 52-60.

Häkkinen, L. V. T., et al. (2003), Seasonal and diurnal variation of geomagnetic activity: Revised Dst versus external drivers, J. Geophys. Res., 108(A2), 1060, doi:10.1029/2002JA009428.

Lal, C. (1998), Solar wind and equinoctial maxima in geophysical phenomena, J. Atmos. Sol. Terr. Phys., 60, 1017-1024, doi:10.1016/S13646826(98)00046-7.

Le Mouël, J. L., E. Blanter, A. Chulliat, and M. Shnirman (2004), On the semiannual and annual variations of geomagnetic activity and components, Ann. Geophys., 22, 3583-3588, doi:10.5194/angeo-22-3583-2004.

Lyatsky, W., and A. Tan (2003), Latitudinal effect in semiannual variation of geomagnetic activity, J. Geophys. Res., 108(A5), 1204, doi:10.1029/ 2002JA009467.

Malin, S. R., and A. M. Isikara (1976), Annual variation of the geomagnetic field, Geophys. J. R. Astron. Soc., 47, 445-457.

Mayaud, P. N. (1978), The annual and daily variations of the Dst index, Geophys. J. R. Astron. Soc., 55, 193-201.

Mursula, K., E. Tanskanen, and J. J. Love (2011), Spring-fall asymmetry of substorm strength, geomagnetic activity and solar wind: Implications for semiannual variation and solar hemispheric asymmetry, Geophys. Res. Lett., 38, L06104, doi:10.1029/2011GL046751.

O'Brien, T. P., and R. L. McPherron (2002), Seasonal and diurnal variation of Dst dynamics, J. Geophys. Res., 107(A11), 1341, doi:10.1029/ 2002JA009435.

Russell, C. T., and R. L. McPherron (1973), Semiannual variation of geomagnetic activity, J. Geophys. Res., 78, 92-108, doi:10.1029/ JA078i001p00092.

Sabine, N. (1856), On periodical laws discoverable in the mean effects of the larger magnetic disturbances. No. III, Philos. Trans. R. Soc. London, Ser. B, 146, 357-374, doi:10.1098/rstl.1856.0016.

Stening, R. J., and D. E. Winch (1987), Night-time geomagnetic variations at low latitudes, Planet. Space Sci., 35(12), 1523-1539, doi:10.1016/ 0032-0633(87)90078-X.

Sugiura, M., and T. Kamei (1991), Equatorial Dst index 1957-1986, edited by A. Berthelier and M. Menvielle, IAGA Bull., 40, Int. Serv. of Geomagn. Indices, Saint-Maur-des-Fosses, France.

Svalgaard, L. (2011), Geomagnetic semiannual variation is not overestimated and is not an artifact of systematic solar hemispheric asymmetry, Geophys. Res. Lett., 38, L16107, doi:10.1029/2011GL048616.

Wardinski, I., and M. Mandea (2006), Annual and semi-annual variations of the geomagnetic field components analysed by the multi-taper method, Earth Planets Space, 58, 785-791. 